\title{
Improving in-Vehicle Display and Control Design for Older Drivers
}

\author{
Jaeheok Ryu ${ }^{1}$, Gyohyeon Song ${ }^{2}$, Seongil Lee ${ }^{2}$, Yoonhyung Cho ${ }^{1}$, \\ Gyouhyung Kyung ${ }^{1}$, Hyungkee Kim ${ }^{1}$, and Kyungkuk Baek ${ }^{2}$ \\ ${ }^{1}$ Package Engineering Team 2, Hyundai-Kia Motors, Korea \\ ${ }^{2}$ System Management Engineering Department, SungKyunKwan University, Korea
}

\begin{abstract}
Guidelines for older driver-friendly automobile interior design have been determined by taking into account older people's physical and cognitive characteristics. Twenty three older people (aged from 54 to 78) and five younger people (from 20 to 29) performed several tasks in actual driving conditions, in which their reaction times and performance errors were recorded. Some design factors were found to be related to older drivers' visibility and controllability. Several design guidelines were proposed in terms of cluster color and font, display location, and HVAC control type. Proposed guidelines are expected to satisfy a wider range of older drivers as these will facilitate automobile interior designs which are fitter to older drivers' visual, cognitive, and manual capabilities.
\end{abstract}

Keywords: older driver, automobile interior, automotive ergonomics, visibility, controllability.

\section{Introduction}

With the increase in the average length of life, Korea is expected to enter an advanced age society in a short time. Generally, older people are those who are aged 65 or older according to WTO (World Trade Organization) and UN (United Nations). The aging society is where the proportion of older people among its total population is more than $7 \%$, whereas the advanced age society is where the proportion is more than $14 \%$, and the post-advanced aged society with more than $20 \%$ of older people in its population. Korea has already entered the aging society as of the year 2000 when $7.2 \%$ of its population aged 65 or older, and recently it has been approaching to an advanced aged society very rapidly. Driving tasks are usually harder for older people due to their decreased physical and cognitive abilities. Hence, the needs and abilities of older drivers should be carefully taken into account when designing automobiles. Studies on older drivers' abilities have been increasing and become vital with the rising number of older drivers involved in car accidents in Europe and North America. Relevant studies led by Toyota, Honda, and Nissan in Japan are more focused on developing welfare vehicles for physically challenged people. Comparatively, studies on older driver-friendly vehicle design in Korea are scarce.

This study attempted to propose automobile interior design guidelines for older drivers in terms of visibility and controllability when interacting with LCD display, cluster gauge, and HVAC (Heating Ventilating, and Air Conditioning) controls. 


\section{Experiment}

\subsection{Process}

First, this study attempted to find older drivers' characteristics and interior design factors through actual driving tests. Second, simulation tests were performed under various design conditions of LCD display, cluster gauge, HVAC and center fascia controls. Lastly, older driver-friendly automobile interior design guidelines were determined. Experiments involved older drivers and large-size sedans.

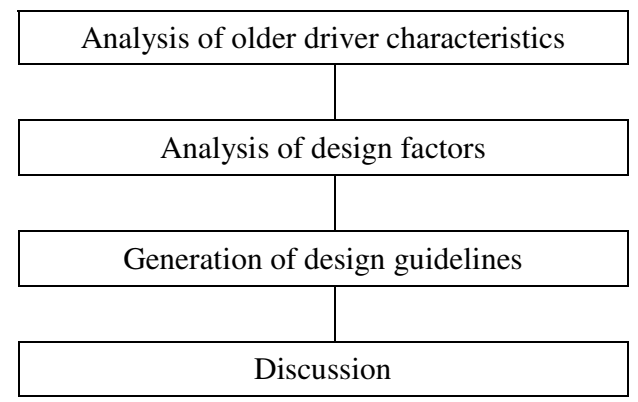

Fig. 1. Research process

\subsection{Methods}

\section{Analysis of Older Driver Characteristics}

Twenty three older drivers (aged from 54 to 78) and five younger drivers (from 20 to 29) performed several tasks and their characteristics and performance data were analyzed. Twenty three older drivers were further classified into two groups, one aged from 54 to 64 and the other aged 65 or older (the latter was referred to as 'the older driver group' in this paper). Experiments consisted of actual driving conditions. Participants performed tasks according to prescribed task scenarios, while driving an experimental vehicle and verbalized their thought using the think aloud method. Their task performance was recorded by 4 pin-hole cameras. Three cameras were mounted

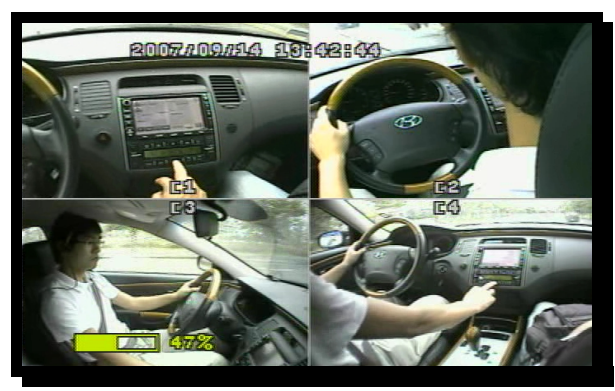

Fig. 2. Recorded actual driving test 
at the driver-side center pillar ('B' pillar) for videotaping the cluster gauge area, the passenger-side front pillar ('A' pillar) for recording driver's reaction, and the left side of the passenger headrest for videotaping the center fascia area. The last one was a headset type for driver's visibility. Fourteen specific task scenarios involve interactions with four interior parts (i.e., LCD display, cluster gauge, and HVAC and center fascia controls) to analyze the older drivers' visibility and controllability. Tasks were performed during day and at night, and reaction times and errors of subjects were also recorded.

\section{Results}

\subsection{Performance in Actual Driving}

First of all, a task was performed to find interior parts that were strongly related to older drivers' visibility and controllability. With drivers' age advanced, longer reaction times and higher error rates were observed when performing tasks in general. In terms of reaction time, old driver group took longer time than those aged from 54 to 64 (7.65s vs. 4.46s). Older people took 3 times longer than younger people. The older driver group showed a higher error rate than the other two groups. In other words, increased reaction time and error rate were observed with age increasing.

The older driver group also showed the longest reaction time and the highest error rate for functional tasks among three age groups. The results were especially influenced by LCD display, cluster gauge, and HVAC controls.

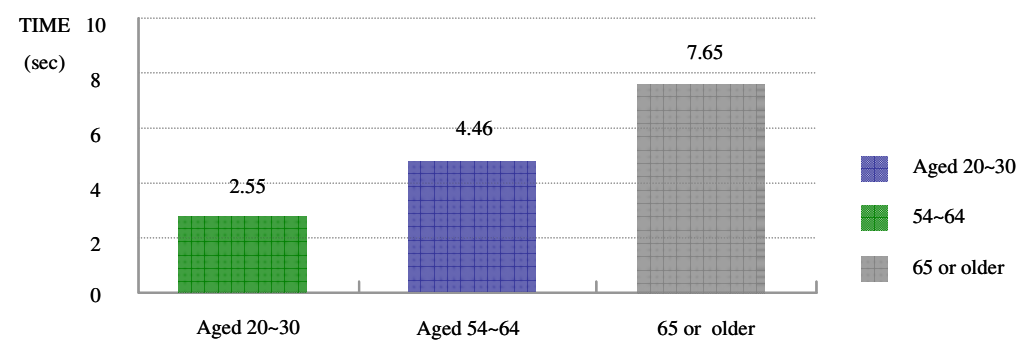

Fig. 3. Task reaction time of three age groups

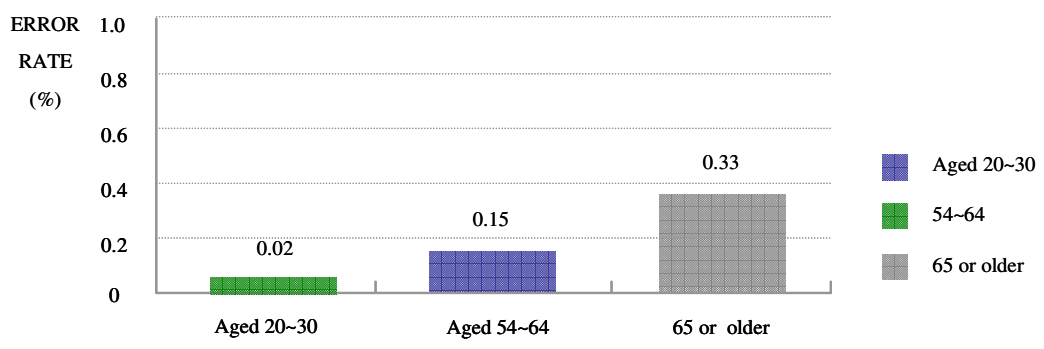

Fig. 4. Task error rate of three age groups 


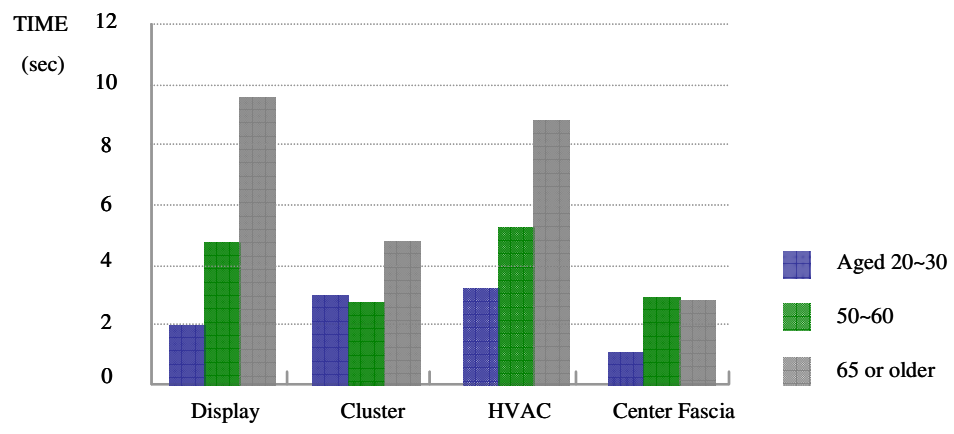

Fig. 5. Task reaction time for function of interior

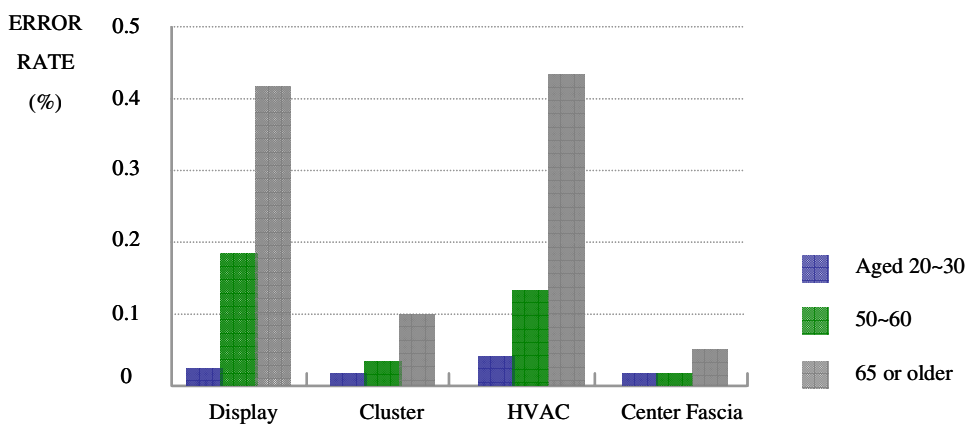

Fig. 6. Task error rate for function of interior

From actual driving tests, differences in driving performance were found according to age and function of automobile interior. Somewhat lower driving performance was observed at night than in daytime.

\subsection{Analysis of Relevant Design Factors}

From actual driving tests, it was found that LCD display, cluster gauge, and HVAC controls influenced older drivers' visibility and controllability. A further experiment was done in the lab environment to determine appropriate levels of design factor of LCD display, cluster gauge, and HVAC controls. To find levels of design factors, the task performance was measured using actual driving task video in the lab setting. It was made up of twelve task scenarios. Task performance was measured in terms of reaction time, error rate, and additionally subjects' satisfaction index was used.

First, a task involving interactions with an LCD display was performed. Four design factors (the display's lateral angle, vertical slope, height, and degree of protrusion) were manipulated. By changing levels of four factors and attaching touch sensors on the display, reaction time and error rate for each task were measured. Satisfaction level was reported verbally as well as recorded on the questionnaire. 


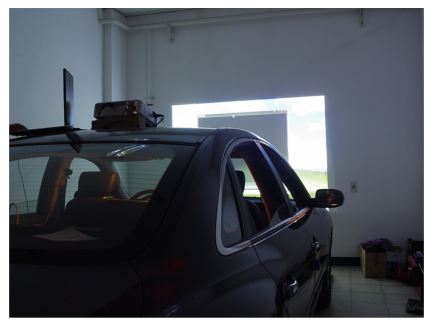

Fig. 7. Simulation test setting

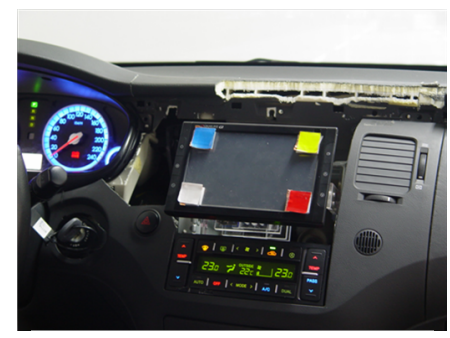

Fig. 8. LCD display test setting

Different task performances were observed between the older driver group and the other two groups when the display's lateral angle was changed. The fastest reaction time and the lowest error rate for the older driver group was when the display was laterally tilted 15 degrees toward the driver, whereas this factor showed little influence on the performance of the remaining groups.

Display slope also led to different task performance between the older driver group and the other groups. The lowest error rate was observed when display slope was set twenty five degrees. When display slope was set fifteen degrees, there was no significant different task performance observed between the older driver group and the other groups, compared to when twenty five degrees were used. However, satisfaction index was a little higher for the older driver group with the display slope of fifteen degrees. Though higher display location was associated with higher satisfaction level, there was little task performance difference between groups. Desirable display height was found to be about $50 \mathrm{~mm}$ higher than the initial location. There was little significant performance difference in terms of display protrusion level. Only for the older driver group, increased protrusion level led to decreased error rate and increased reaction time.

A second simulation test was performed with HVAC controls. HVAC controls were classified into three types (i.e., mode, dial, and switch types). The mode type had different ventilation direction controls than the switch type; The latter comes with one-switch, one-direction controls, while the former had one-switch, multi-direction controls. LED was added to the mode type HVAC control and traditional colorcoding was used for temperature (i.e., Red for increasing temperature, and blue for decreasing temperature). HVAC control type was associated with different performance. A faster reaction time and the lowest error rate were found when using the switch type HVAC control, which was rated most intuitive by the participants. 

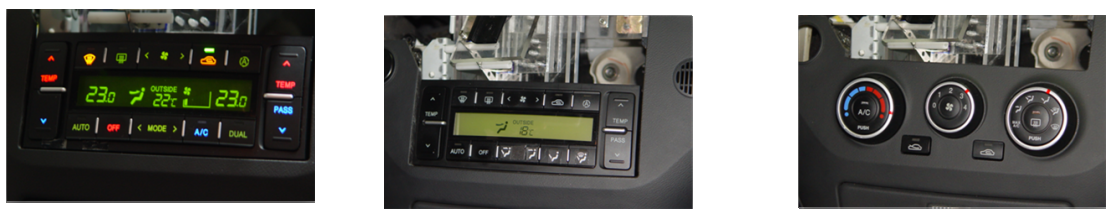

Fig. 9. Three HVAC control types (mode, switch, and dial types)

Lastly, cluster gauge visibility was tested using a total of eight different cluster films that varies by color (4 levels: white, blue, red and orange) and font ( 2 levels: type A and B). Type B font came from a bestselling large sedan in the USA.
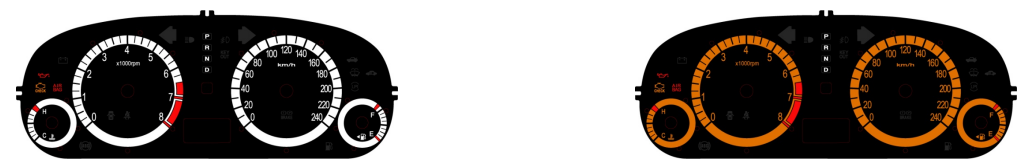

Fig. 10. Examples of cluster prototypes used (white and orange background colors)

Generally, a letter can be designed with a different width, space between letters, thickness, and font type and so on. The letter width and space between letters used for the car B were $3 \mathrm{~mm}$ and from 0.5 to $1 \mathrm{~mm}$, respectively, while those of the car A were $4 \mathrm{~mm}$ and $0.5 \mathrm{~mm}$. Letters of the car A cluster were hence thicker and wider than those of the car B.

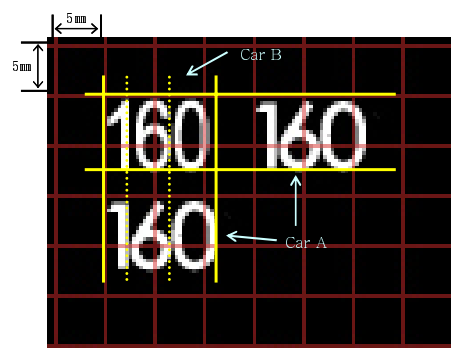

Fig. 11. Comparison of cluster gauge letters

In conclusion, increased reaction time and error rate and decreased satisfaction were observed when the red-colored cluster was used for the older driver group. Increased satisfaction was associated with the white-colored cluster for the older driver group, and with the white-colored and the blue-colored cluster for the other two groups. And in the case of the older driver group, faster reaction time was observed with the car A cluster. Letter thickness and wide space between letters used in the car A cluster seems to be associated with this result. Additionally, some subjects misperceived ' 6 ' used in the car B cluster as ' 8 '. 


\subsection{Design Guidelines}

1. LCD display height is recommended to be placed about $50 \mathrm{~mm}$ higher than considered for the car B, and the display should be slanted in fifteen to twenty five degrees. Visibility and controllability can be further improved when the display could be tilted 15 degrees toward the driver.

2. HVAC controls should be designed using proper switch types which are intuitive the older drivers. Additionally, adding the red and blue color on the switch improves visibility and controllability.

3. In the case of the cluster gauge, white and/or blue color, and letter thickness of $1 \mathrm{~mm}$ are recommended. Also, the length and width ratio of a letter should be set to 5:7 to improve readability.

\section{Conclusion}

Several guidelines for older driver-friendly automobile interior design have been determined by taking into account older people's physical and cognitive characteristics. Some design factors related to older drivers were determined through the actual driving test. Design guidelines for LCD display, cluster gauge, and HVAC controls could be proposed through the simulation test. Further study will be needed with more participants. The results from the current study will contribute to increasing the older drivers' safety and convenience, and will help to meet their needs.

\section{References}

1. Wickens, C.D., Hollands, J.G.: Engineering Psychology and Human Performance, 3rd edn., p. 573. Prentice Hall, Englewood Cliffs (1999)

2. Wickens, C.D., Gordon, S.E., Yili, L.: An Introduction to Human Factors Engineering, 750 p. Prentice Hall, Englewood Cliffs (1997)

3. Herriotts, P.: Applied Ergonomics 36, 255-262 (2005)

4. Smith, D.: Human factors and aging; an overview of research needs and application opportunities. Human Factors 32(5), 509-526 (1990)

5. Misugi, K., Kanamori, H., Koyama, N., Atsumi, B.: Toyota's Program for Universal Design in Vehicle Development. In: Designing For The 21st Century III, Brazil (December 2004) 